\title{
THE HYDROCEPHALUS OF POLIOMYELITIS *
}

\author{
JOSEPH G. REGAN, M.D. \\ BROOKLYN
}

So prominent is this symptom of hydrocephalus in the pathologic and clinical picture of poliomyelitis, and so important is it from the viewpoint of treatment, that it seemed advisable to speak of it at length, and at the same time to show the value of Macewen's sign in its recognition.

In order to adequately discuss the hydrocephalus of poliomyelitis, it is necessary first to understand the pathologic basis for the symptom. Recent researches have shown that the earliest microscopic change that is found in the cord and brain is hyperemia and the collection of small mononuclear cells in the perivascular lymph space of the blood vessels of the leptomeninges. A sheath of cells is thus often formed completely encircling the vessel for a considerable length. This sheath may be so dense that the lumen is encroached on and the circulation is partly obstructed. Draper, Peabody and Dochez ${ }^{2}$ think there may be some effect either toxic or mechanical which acts on the intimal lining of the blood vessel walls, for hemorrhages, minute or extensive, are frequent findings, and one of the pronounced features of most cases is the extensive edema. Wickman, ${ }^{2}$ in his classical monograph, points out that edema may be so great as to appear to the naked eye like a general softening of the cord, and also that the brain may be so edematous that the dura is rendered tense and the convolution flat. He says that microscopic examination shows the walls of the veins to be more infiltrated than those of the arteries. Romer ${ }^{3}$ also notes the intense edema often encountered and the greater tendency for the infiltration to involve the veins. In the twenty odd cases of poliomyelitis necropsied at the hospital during the epidemic of 1916 by Regan and Rosenthal; there has been likewise a generalized edema, more

* Submitted for publication Nov. 24, 1917.

* From the Kingston Avenue Contagious Disease Hospital, Department of Health, New York City.

* The writer wishes to express his appreciation of the cooperation of Dr. Robert J. Wilson, Director of the Bureau of Hospitals, Department of Health; also to the following members of the visiting staff: Drs. L. C. Ager, E. S. Dalton, J. P. Pendleton, S. Feldstein, M. B. Gordon, W. Truslow and J. F. Crawford.

1. Draper, G., Peabody, F. W., and Dochez, A. R.: Monograph 4, Rockefeller Institute for Medical Research, New York, 1912, p. 20.

2. Wickman, Ivan: Nervous and Mental Diseases, Monograph 16, New York, 1913, p. 14.

3. Romer, P. H.: Epidemic Infantile Paralysis, New York, 1913, p. 104. 
markedly so in some cases than in others. Thus macroscopically the cord would usually be bulging between the strands of pia mater which covered it, and on cross section had a moist translucent appearance, and would often evert over the edge of the membrane as a collar. The consistency was softer than normal. Not infrequently the brain was so closely crowded against the calvarium that the convolutions of the superior aspect were decidedly flattened in places. In such instances the ventricles have been enlarged and evidently during life were distended with fluid. As in the case of the cord, the cut surface of the brain had a moist, translucent appearance, and was definitely of a softer consistency than normal, so that it was often a very difficult task to remove it intact from its bony envelope.

That edema should arise from diverse causes is not surprising when consideration is given to the particular type of pathologic alteration that occurs in this disease. Several factors especially predispose to the supervention of such a condition. Thus, the return flow of lymph from the tissues of the cord and brain, which probably normally passes in part along the perivascular sheaths of the blood vessels, is partially, and, in some cases, almost completely interfered with by the infiltration of round cells. Moreover, this infiltration being morc intense in the veins than in the arteries, and the lumen of the former vessels being more easily obstructed by changes within their walls, owing to a less rigid structure, the venous return flow is more hindered than the arterial inflow, with a resultant tendency toward venous stasis and hence toward edema. Then, again, there may be a greater permeability to the intima of the vessels, which allows of serous exudation. It is, moreover, by no means improbable that the generalized inflammatory reaction of the nervous system may involve the pacchionian bodies to a sufficient extent as to interfere materially with the excretory function which they are normally supposed to exericse.

In addition to the edema of the tissues, there is present in a high proportion of the patients an actual increase in the quantity of spinal fluid in the subarachnoid spaces and in the ventricles of the brain. This is obvious before death, by the large quantities of fluid commonly removed by lumbar puncture, and postmortem, by the increased amount present in the subarachnoid spaces and ventricles, especially when the necropsy is performed a very short interval after death. A similar observation postmortem has been made by Wickman and by Romer. The choroid plexuses of the ventricles, especially the lateral, which are, according to our present knowledge, the secretory organs for the production of spinal fluid, are composed of anastomosing arteries and veins, and no doubt are intimately connected with hypersecretion. Poliomyelitis is accompanied, as we know, by an increased hyperemia of the brain, and this hyperemia involves naturally the 
blood vessels of the choroid plexus. In addition to this, Draper ${ }^{4}$ claims that the perivascular infiltrations of the blood vessels may also involve those of the plexus. This being so, and the veins of the choroid probably being more concerned than the arteries, there is a distinct tendency toward interference with the venous return flow despite the active arterial supply. As a result of these various factors, hypersecretion results, producing an increased intracranial pressure, which reflexly causes a rise in the arterial and venous pressure throughout the cerebral circulation. Normally the excretion of the cerebrospinal fluid through the sinuses seems to depend on the fact that the intracranial pressure is slightly higher than the pressure in the veins. With the pressure in the veins raised equally with the intracranial pressure, excretion through the pacchionian bodies into the sinues is greatly retarded if not almost arrested, and further accumulation of cerebrospinal fluid occurs in the ventricles and subarachnoid spaces. This, in conjunction with the generalized edema of the tissues, presents a typical picture of hydrocephalus and gives rise to the clinical symptoms which we shall now describe, after classifying them as follows:

1. The hydrocephalus of the onset, including the preparalytic and early part of the paralytic stages.

2. The hydrocephalus persisting after the first week of the disease and comprising three types: (a) a mild form commonly encountered in which there is only a slight increase in fluid and in which symptoms are practically absent; $(b)$ a more severe form accompanied by symptoms of pressure; $(c)$ a very severe type, more or less insidious in onset, indefinite in physical signs, and associated with evidences of progressive emaciation.

1. The Hydrocephalus of the Onset.-As has been described under the pathologic picture of the disease, the onset of the meningeal phase of poliomyelitis is accompanied by a marked increase in the spinal fluid content of the subarachnoid space. Coincident with this, symptoms of acute hydrocephalus appear. The child becomes drowsy or stuporous, the pupils dilated, and react rather sluggishly to light; irritability is often marked; the respirations and pulse become rapid and sometimes irregular; vasomotor flushings of various parts of the body appear. Ataxia, headache, rigidity of the neck and profuse sweating may also occur and be due in part to the same cause. All these symptoms are strikingly alleviated by lumbar puncture, and large amounts of fluid, 40 to 80 c.c., may be obtained under considerable pressure. Even after paralysis has supervened these symptoms often still persist unless relieved by puncture. It is, moreover, noteworthy that paralytic phenomena of the early stages may often be, in part, due to the edema

4. Draper, G.: Acute Poliomyelitis, Philadelphia, 1917, p. 35. 
and the hydrocephalus, for not infrequently there will be definite improvement following relief of pressure. It is in such instances that Macewen's sign is of the utmost value, for by its use the suspected group of symptoms can be definitely interpreted and suitable treatment instituted. ${ }^{5}$ No doubt it is to be found present to some degree in practically all cases of poliomyelitis in the preparalytic stage.

2. The Hydrocephalus Persisting After the First Week of the Disease.-(a) The condition may be so mild that practically no symptoms are noticeable, yet often a slight Macewen sign will be present and lumbar puncture will yield an increased quantity of spinal fluid under slight pressure. This is the usual type.

(b) In other cases there may be distinct evidences of pressure, as shown by persistence of the dilated pupils, the headache, the drowsiness or stupor, rigidity of the neck and the irritability when disturbed. The headache, the ataxia, the rapid respirations, the pulse, and the elevated temperature may all remain for an unusually long period, and the cause be determined by the presence of a positive Macewen sign or of a bulging fontanel. One or two lumbar punctures, with the removal of sufficient fluid to bring the pressure to normal-about 20 drops a minute-will often be sufficient to dissipate the symptoms and not infrequently materially improve the prognosis with regard to paralysis.

We have occasionally encountered cases running a persistently high temperature, and a moderately increased pulse and respiration, in the second or early part of the third week of the disease, with no physical findings to account for the febrile phenomena, in which the performance of a lumbar puncture would often be followed by the subsidence of the symptoms (Cases 3 and 4). Sophian, ${ }^{6}$ in his clinical study of epidemic meningitis, noted cases of a somewhat similar character and attributed them also to hydrocephalus.

Patients with paralysis of the intercostal muscles alone or with slight involvement of the diaphragm not infrequently present periods in which the breathing gradually becomes more dyspneic and labored, the diaphragmatic action more jerky and irregular, the pulse rapid, the pupils rather dilated, and a nervous apprehension and irritability is manifest. At the same time perspiration often is notably profuse and Macewen's sign prominent. The removal of a sufficient quantity of spinal fluid will usually be followed by the disappearance of the alarming symptoms.

5. It seems extremely probable that an examination of the eyegrounds would also be of considerable value in establishing the diagnosis of hydrocephalus, especially if the condition had been existent for several days.

6. Sophian, A.: Epidemic Cerebrospinal Meningitis, St. Louis, 1913, p. 138. 
(c) A persistent hydrocephalus of this type is the most serious and the most hopeless of the different forms, but luckily it is not common. We have only encountered it in a small number of our cases. It usually supervenes at times in patients with extensive paralysis, involving all four extremities and the intercostal muscles. These children were usually desperately ill during the first week or two of the disease, and although passing through the danger period, showed no signs of improvement either in their general condition or in their paralysis. On the contrary, a decided loss of weight and rapid shrinking of the subcutaneous tissues was noticeable. The loss of tissue rapidly assumed alarming proportions, despite the ingestion of large quantities of food (for the appetite is sometimes ravenous, owing no doubt to central involvement of the vagus nerve). Eventually, extreme emaciation appears, and trophic changes occur in the skin, especially over bony prominences, such as the buttock or the back of the head, heels, etc., with the production of deep ulcers which may extend to the bone and show no tendency to heal. The mentality of the child gradually becomes dull and he ceases to take any interest in his surroundings, and finally dies in a state of acute inanition. Lumbar puncture is entirely useless in such cases, as the edematous condition of the brain seems to have assumed a subacute or chronic course with no tendency to subside.

Heiman and Feldstein, ${ }^{7}$ and later, Sophian, ${ }^{8}$ in their excellent studies of epidemic meningitis, describe very similar cases, and, indeed, so close is the resemblance of most of the hydrocephalic symptoms in the two diseases that we have modified somewhat our classification after that which Koplik ${ }^{9}$ used in his scientific clinical consideration of the hydrocephalus of meningococcus meningitis.

\section{REPORT OF CASES}

CASE 1.-Illustrating chronic hydrocephalus of poliomyelitis.

No. 2195 , G. D., aged 5 years; ill three days when admitted to the hospital, August 4; discharged Oct. 3, 1916.

The condition on admittance was poor. The child was fairly well developed and nourished, but rather oversized for his age. He laid in a stuporous condition, out of which, however, he could be somewhat aroused. Macewen's sign was a markedly positive one; the neck was rigid anteroposteriorly, less so laterally. The pupils were dilated, equal, and reacted to light. Breathing was shallow, abdominal, with slight costal movement of the lower ribs, evidently due to the diaphragm, the intercostal muscles being completely paralyzed. There was complete flaccid paralysis of both upper and lower limbs, with absence of reflexes. The heart sounds were of fair quality, but the pulse was rapid and of very poor volume. The abdominal organs showed no apparent alteration.

7. Heiman, H., and Feldstein, S.: Meningococcus Meningitis, New York, 1913, p. 204.

8. Sophian, A.: Epidemic Cerebrospinal Meningitis, St. Louis, 1913, p. 81.

9. Koplik, H.: Quoted by Heiman and Feldstein (Footnote 7), p. 197. 
Lumbar punctures were performed on August 5, 11, 16 and 19, with the withdrawal of, respectively, 20,32, 22 and 20 c.c. of clear fluid in the order named. The spinal fluid of August 11 showed no increase in cells; a few mononuclears were present, however; albumin, ++ ; globulin, ++ ; Fehling's test, +++ ; no organisms on smear or culture. Following the first puncture there was a slight improvement in the stupor, the child became apathetic and developed a ravenous appetite, crying more or less continually in a very feeble and indistinct voice for food. Despite the consumption of large quantities of nourishment the fulness of the subcutaneous tissues soon disappeared, the overlying skin becoming wrinkled, and over the bony prominences showed trophic changes. Thus, in the region of the elbows, knees, heels and buttocks there appeared large areas of discolored, unhealthy though not ulcerated skin. An examination of the eyegrounds by our ophthalmologist, Dr. Berry, August 16, showed a marked retinal congestion. August 18, the child developed a severe apthous stomatitis, with the formation of creamy white plaques, almost $3 \mathrm{~cm}$. in diameter, on the edges of the tongue, the insides of the lips and cheeks and along the gums. Mastication was rendered difficult for a few days, but the condition soon passed off. The child still remained in a condition of apathy, lying for hours in the same position, his eyes wide open, with a vacant, or, at times, anxious expression on his face, which had now assumed the shrunken appearance of an aged person. The pupils were widely dilated but reacted to light. The abdomen was sunken,

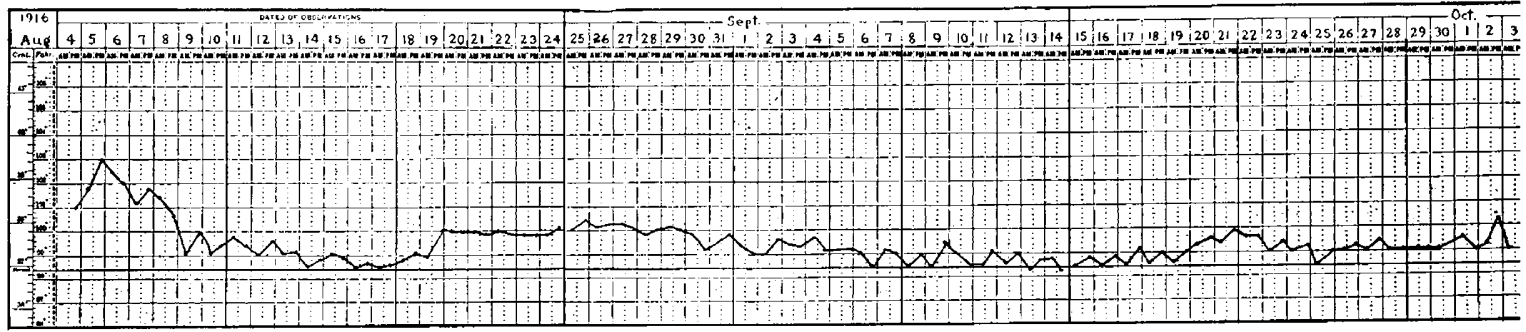

Fig. 1.-Temperature curve, G. D., Case 1 (No. 2195).

giving it a scaphoid appearance; the upper chest was constricted and narrowed, consisting only of the bony framework and the overlying skin. The lower chest was flaring, due to the unopposed action of the diaphragm and the thoracic and abdominal pressure during inspiration. The legs and arms became extremely emaciated; indeed, to such an extent that one hesitated to move him for fear of spontaneous fractures or dislocations. For hours at a time the child would cry, or rather whine, in a feeble voice. Not infrequently his actions and the few words he spoke that were understood indicated a clouded mentality and that he was suffering from hallucinations. The temperature and pulse remained slightly elevated and were subject to irregular daily variations (Chart 1 ). There was complete incontinence of urine and feces. The patient was discharged October 3 , and the subsequent history was not obtained.

Remarks: This case showed the rapid and extreme wasting, which is also commonly seen in the chronic hydrocephalus of meningococcus meningitis, and this despite the ravenous appetite of the patient. The altered mental condition is also especially worthy of notice.

CASE 2.-Also illustrating chronic hydrocephalus.

No. 1778, H. O., aged 4 years; taken ill two days before admission; admitted July 24, died Sept. 2, 1916. The condition on admission was very poor, pulse barely perceptible, extremities rather cold, some cyanosis of skin and mucous membranes. The child was in a condition of deep stupor; could not be aroused 
and was entirely unconscious of her surroundings; Macewen's sign was markedly positive; pupils dilated but reacted sluggishly to light; neck was flaccid; respirations were shallow, slow, and costo-abdominal. Evidently the intercostals were completely paralyzed and the diaphragm was working poorly. The knee jerks and plantar reflexes were absent. There was no Kernig sign, nor was there any polyneuritis. Paralysis involved almost the entire body-both uppers and lowers, back, neck and intercostals-and was flaccid in type. In addition, the cranial nerves were concerned; thus, there was a right internal strabismus, evidently due to paralysis of the abducens, a partial paralysis of deglutition, due to involvement of the glossopharyngeal or vagus nerves, and probably a laryngeal paralysis due to affection of the laryngeal branches of the vagus. A direct laryngoscopic examination was impossible owing to a spastic condition of the jaw muscles-"trismus"-which also rendered feeding difficult at first, except by gavage and nutrient enema. The heart sounds were of good quality and distinct, but the pulse was of very poor volume. The abdominal organs were negative; the nutritional condition and development fair; the conjunctiva injected, and there was a slight mucopurulent discharge.

Spinal puncture was performed on admission and 50 c.c. of clear fluid under marked pressure were removed. Punctures were performed on the 26th, 27th, and 31 st, and 45,40 and 20 c.c. of fluid were removed, in the order named. The examination of the spinal fluid of July 26 showed albumin +++ , globulin +++ , Fehling's +++ ; cells not specified; no organisms on smear

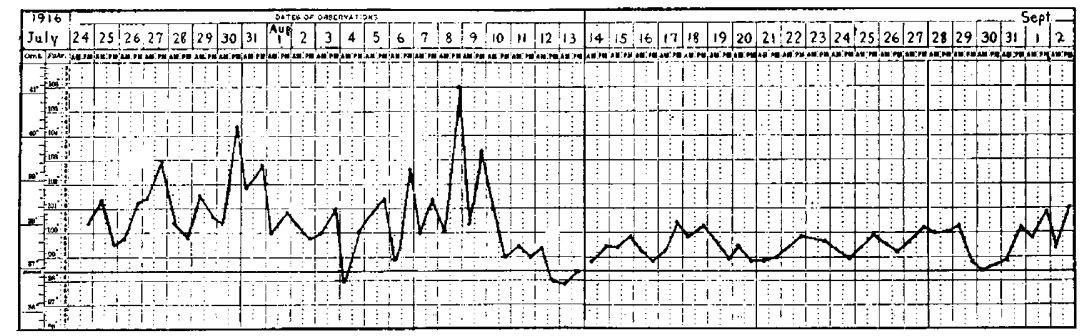

Fig. 2.-Temperature curve, H. O., Case 2 (No. 1778).

or culture. That of July 27 showed a slight increase in cells, 99 per cent. large mononuclears; albumin + , globulin + , Fehling's test +++ ; no organisms on smear or culture. There was a slight improvement in the stupor after the second puncture, and this continued, but was only to a relatively unimportant degree, the patient still lying in an apathetic state, but showing some desire for food. The respiration was less dyspneic, deglutition was somewhat easier and the pulse improved following the punctures. A rapid emaciation set in, however, almost from the start, and it was out of all proportion to the loss entailed by the diminished ingestion of food. This symptom soon became so pronounced that the patient's body was composed only of the bony framework and skin; it might truly be said that the child was a living skeleton. The upper and lower limbs became flexed at the elbows and the ankles; pressure or trophic changes occurred in the skin, over the buttock and at the back of the head. In the latter position a large indolent ulcer, comprising the entire back of the head, was formed, with the bone devoid of periosteum as its base. There was complete incontinence of urine and feces. The patient laid in this condition day after day, until death occurred in the sixth week of the disease, with the symptoms of respiratory paralysis.

Remarks: In this case the partial paralysis of deglutition and the "trismus" rendering feeding difficult at first, would of course account for some wasting. But the emaciation appeared from the start, and was too extreme to be due to this cause alone. Moreover, there were other cases equally as difficult to 
feed, who in the same length of time showed not nearly the same degree of inanition. There was also present a mental deterioration very similar to that noted in the previous case.

CASE 3.-Illustrating persistently high temperature, with no physical signs to account for it except a condition of distinct hydrocephalus.

M. W., No. 2559, aged 3 years; ill two days on admission, Aug. 12, 1916. Discharged Oct. 5, 1916. Physical examination on entrance, showed a well developed and well nourished child, very irritable, and desiring to be left alone; crying frequently during the examination; evidently hyperesthesia was intense. The pupils were equal, rather dilated, and reacted to light. Macewen's sign was present and rather marked. The neck was not rigid but flaccid, and did not support the head. The patellar reflexes were absent; plantars were present but sluggish. There was a modified Kernig sign in both legs. The breathing was costo-abdominal in type, but somewhat shallow. Both legs were partially paralyzed, also the neck. The back was rigid, and there was a right internal strabismus present. The heart and lungs as well as the abdominal organs showed no detectable pathologic change. The temperature was $101 \mathrm{~F}$. on admission and remained persistently at the same level, except for variations of one or two tenths of a degree, until August 17, and this despite the absence of obvious physical signs to account for it (Chart 3). Owing to the presence of symptoms pointing to hydrocephalus a lumbar puncture was per-

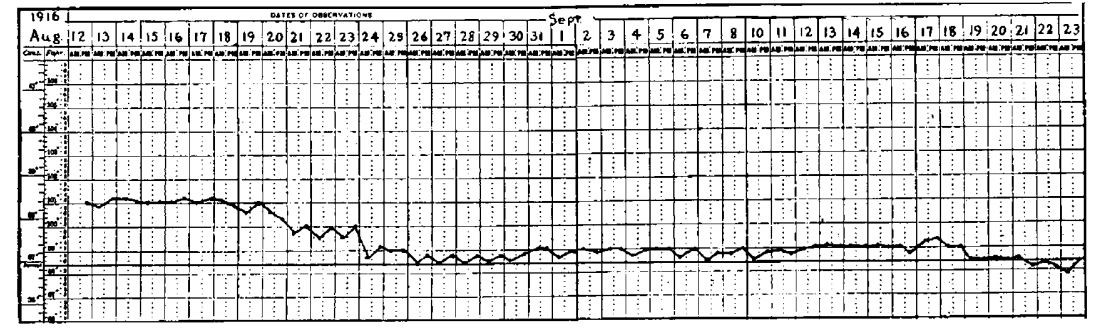

Fig. 3.-Temperature curve, M. W., Case 3 (No. 2559).

formed on this date with the withdrawal of 28 c.c. of clear fluid under pressure, followed by the injection of 10 c.c. of normal horse serum. As the temperature remained still at the same level on the following day, the procedure was repeated and 20 c.c. of slightly opalescent fluid was withdrawn. Following this withdrawal the temperature began to fall by lysis, reaching the normal August 26. Accompanying this there was a fall in pulse rate and a general improvement in the patient's condition, together with a subsidence of the meningeal symptoms-hyperesthesia, irritability, Macewen's sign, Kernig's signand the breathing became deeper. The paralysis improved soon to such an extent that the child could sit up in a chair. Having once attained the normal point, the temperature continued at $99 \mathrm{~F}$. or thereabouts, throughout the rest of the time spent in the hospital.

Remarks: Hydrocephalus may cause a similar continuous elevation of temperature in other diseases. We have encountered it in complicated cases of measles, and in cases of epidemic meningitis, and it has also, in these instances, yielded to rachiocentesis.

CASE 4.-J. W., aged 4 years, No. 2560, was admitted August 12 and discharged Sept. 5, 1916; ill two days before entrance to the hospital. The patient was a sister of the patient, Case 3. The clinical symptoms and history were very similar with the exception that in this case the temperature remained persistently at $103 \mathrm{~F}$. for three weeks. Two lumbar punctures were performed, with the removal of 25 c.c. and 30 c.c. of clear fluid on August 14 and 17, 
respectively, and while the temperature subsided somewhat after the 17 th and the meningeal symptoms improved, neither disappeared till two weeks later. The examination of the spinal fluid of August 14 showed fibrin present, slight increase in cells, 98 per cent. mononuclears, albumin, ++ ; globulin, ++ ; Fehling's test, +++ ; no organisms on smear or culture; while that of August 17 showed the presence of fibrin, slight increase in cells; 99 per cent. mononuclears; albumin, + ; globulin, + ; Fehling's test, +++ ; no organisms.

Remarks: It is probable that had the punctures been repeated persistently, convalescence would have been considerably hastened.

CASE 5.-Illustrating persistent hydrocephalus, with the symptoms of toxemia, and a prolonged elevation of temperature as zell as of a marked polyneuritis but without extreme emaciation.

J. E., No. 3074, ill four days before entrance; admitted Sept. 3, 1916; discharged Oct. 24, 1916. On admission the child was fairly well developed and nourished, but was acutely and desperately ill. He was extremely irritable, crying on the least handling; evidently hyperesthesia was marked. At the same time if he was not disturbed he became drowsy and laid in a condition of light stupor. Macewen's sign was a markedly positive one. The pupils were dilated, even, and reacted to light. The neck muscles were flaccid. The breathing was costo-abdominal, but shallow, the costal movement comprising only the lower ribs and being evidently associated with the motion of the diaphragm, and not due to the intercostal muscles. The knee jerks were absent, the plantar reflexes were exaggerated and the abdominal muscles were normal on both sides. There was a modified Kernig reaction present which was accompanied by a great deal of pain. The child was unable to sit or stand. Paralysis involved the muscles of the neck which were flaccid, the intercostals, the right upper and both lowers, which were rigid, and the left upper extremity, which was flaccid.

There was marked hyperesthesia along the spine and polyneuritis along the posterior aspect of both legs and thighs. At times the child's respirations became rather jerky and irregular, and the skin somewhat cyanotic. The pulse was poor in volume and was rapid, but the heart sounds were of fair quality.

On September 4 the first lumbar puncture was performed, 20 c.c. of bloody fluid removed and 16 c.c. of serum injected. September 6 the child's condition was slightly improved, he was less drowsy, the respirations were somewhat fuller and less jerky, and the color and pulse better. September 8, 16 c.c. of convalescent serum were given by intramuscular injection. The blood count before the injection was: total leukocytes per c.mm., 6,900; polynuclear neutrophils, 61 per cent.; while on September 9 the count was 11,300 whites and 72 per cent. polynuclears; on September 10, 12,700 whites and 70 per cent. polynuclears. September 9 the second puncture yielded 28 c.c. of slightly opalescent fluid under pressure. The cells were moderately increased-97 per cent. mononuclears-the albumin, + ; globulin, + ; Fehling's test, ++ ; no organisms on smear or culture.

Between September 9 and 14 there was slight but continuous improvement, the child's general condition was better, he was less extremely irritable, cried less when handled, his extremities became less rigid and he began to use them slightly; the respirations became easier and deeper, the pulse improved. Macewen's sign became less marked but was still present to a moderate extent. The elevated temperature persisted, however, ranging around $101 \mathrm{~F}$. There were no other physical findings in the lungs, heart or abdominal organs to account for it. Between September 14 and 18 the symptoms of irritability, more labored respiration, more marked Macewen's sign, again became conspicuous and another lumbar puncture was performed September 18, this time with the removal of 20 c.c. of clear fluid under pressure. The fluid showed a slight increase in cells, all mononuclears; albumin, + ; globulin, $+;$ Fehling's test, +++ . The symptoms were temporarily alleviated, but September 23 the procedure had to be repeated and 30 c.c. were obtained under marked 
pressure. Examination showed moderate increase in cells, 98 per cent. mononuclears; albumin, + ; globulin, + , Fehling's test, ++ . September 25 the patellar reflexes had reappeared in the left leg, the rigidity had almost disappeared from the legs and arms, the breathing was deeper, the child's general condition was better, he was brighter and not so irritable, but it was noticeable that there had been a considerable loss of weight since admission. The elevated temperature still remained and continued so until discharge, with nothing other than the changes in the central nervous system to account for it, Macewen's sign was present throughout the period. The excitable, irritable, and "on edge" disposition, the polyneuritis of the lower limbs, remained to some extent, though improving slowly in conjunction with the paralysis.

Remarks: In some cases of poliomyelitis, like this last, for example, it would appear that the infection of the tissues of the central nervous system by the virus of the disease was of a persistent and deep seated character, not running its course in a few days or weeks, but extending over a long period of time and giving rise to the symptoms we have recorded. Whether the hydrocephalus is the predominant feature of these cases, or whether it is merely secondary to persistent infection or toxemia is difficult to decide. It is in such cases as these that one might well expect the causative agent of the disease to be given off in the nasopharyngeal secretions for several months after the acute petiod of the disease.

CASE 6.-Illustrating a very severe and almost fatal type of hydrocephalus, concomitant with the onset of the disease, but subsiding rapidly following rachiocentesis.

No. 3003, B. H., aged $8 \frac{1}{2}$ months; ill five days before admission, August 28; discharged Oct. 16, 1916. Examination on admission at $7 \mathrm{p} . \mathrm{m}$., showed the child extremely cyanotic; the respirations were very shallow and somewhat stertorous and extremely irregular, being interrupted by periods of complete cessation of breathing for as long as two minutes. During these periods the cyanosis became extreme and artificial respiration was necessary. Macewen's sign was marked despite the presence of a bulging fontanel. The pupils were constricted and the eyelids tightly closed. The child was deeply stuporous. Apparently there was paralysis of both lower extremities present, but it was difficult to determine as the child was in a condition of extremis. Patellar, plantar, biceps, and abdominal reflexes were apparently absent. The respirations were abdominal in type, but very shallow.

At 9:30 p. m., a lumbar puncture was performed and 68 c.c. of clear, transparent fluid was removed under marked pressure, and 14 c.c. of horse serum were injected by the gravity method.

During the withdrawal of fluid and immediately following it for one and a half to two hours, the child had several attacks of severe and prolonged suspension of all respiratory movements, often for as long as one to three minutes at one time. At such periods there was generalized cyanosis and complete disappearance of radial pulsation. Artificial respiration was urgently needed during these periods to keep the patient alive. Between $11 \mathrm{p}$. m. and 3 a. m., August 29 , the condition remained still one of "extremis," and oxygen was used almost continuously during each spell of apnea. It was given by Dr. Meltzer's apparatus for the administration of oxygen under pressure and quickly relieved the cyanosis. Hypodermic injection of atropin sulphate, $1 / 360$ grain, and camphor, 2 grains, were also resorted to during this interval. After 3 a. m. the child improved. The spells of apnea disappeared, the cyanosis became less and the breathing deeper, while at the same time the radial pulse became of much better volume. The stupor lessened and the child began to take nourishment.

On the following day, August 29, a more complete physical examination was made. It gave the following findings:

The child was very well developed and nourished; rather drowsy; fontanels slightly tense but not bulging; slight rigidity (anteroposterior) of the neck; 
pupils constricted, but reacted to light; left internal strabismus present; respirations were purely abdominal, but while shallow, were fairly regular, and there was no apnea. There was apparently no definite paralysis, except the strabismus. Patellar and plantar reflexes were normal. There was no Kernig's sign. The heart was rapid but the sounds were of good quality. The lungs showed some areas of bronchovesicular breathing, but no râles. The abdomen was distended. The liver was slightly enlarged, extending two fingers' breadth below the costal margin. The improvement in the child's condition, compared to the previous night, was simply astonishing. From the grave, almost moribund state on admission, he was transformed within twenty-four hours into a convalescent and merely by one spinal puncture. An examination of the spinal fluid showed a slight increase in cells; 98 per cent. mononuclears; albumin, +; globulin, + ; Fehling's test, ++ .

August 31 the respirations increased to 40 and the temperature increased slightly. The child coughed frequently and there were some rather fine râles scattered in a few areas on both sides of the chest. This subsided in three days and convalescence was rapid and complete, the child being discharged with no residual paralysis except the slight strabismus.

Comment: This case shows an extreme form of hydrocephalus of the very acute stage, which would probably have terminated fatally but for the timely removal of fluid. The noteworthy thing is the rapid transition of the patient from a condition of extremis to convalescence in a few days.

\section{SUMMARY}

Researches pursued on the pathology of poliomyelitis have shown that edema is one of the prominent characteristics of the disease. Several factors especially predispose to such a condition: thus, the return flow of lymph from the issues of the cord and brain is interfered with by the cellular infiltration of the perivascular sheath of the blood vesscl wall, while at the same time the veins being more infiltrated than the arteries and their lumen more easily obstructed, due to a less rigid structure, the venous return flow is more hindered than the arterial inflow, with a resultant tendency toward venous stasis and hence toward edema. These two factors in conjunction with the congestion accompanying the early stage of inflammation explain in part the edema. There may be concerned also some toxic effect acting locally on the blood vessel wall.

In addition to the edema of the nervous system, there is also an actual increase in the quantity of spinal fluid in the subarachnoid spaces and in the ventricles of the brain. This is obvious before death by the large quantities commonly obtained by lumbar puncture and postmortem by the increased amount found present. There is probably coincident with the congestion of the brain a hypersecretion of cerebrospinal fluid by the choroid plexuses and at the same time the excretion into the sinuses through the pacchionian bodies is temporarily unable to handle the increased amount produced.

Therefore it is not surprising that this condition of acute nonobstructive hydrocephalus should give rise to certain definite clinical symptoms. According to the type of symptoms and the time they 
persist, we have found it advisable to divide the hydrocephalus of poliomyelitis into the following forms:

1. That of the onset (including the preparalytic and early paralytic stages).

2. That persistent after the first week of the disease and comprising three varieties: $(a)$ a mild form commonly encountered in which there is only a slight increase in fluid and in which symptoms are praccally absent; $(b)$ a more severe form comprising various degrees, in which there are distinct signs of pressure; $(c)$ a very severe type, more or less insidious in onset, indefinite in physical signs, and associated with evidences of progressive emaciation.

The symptoms of these various types are described under their suitable headings in the preceding text, and suitable case reports are included to amply explain these descriptions. ${ }^{10}$

Macewen's sign provides a definite means by which these symptoms of hydrocephalus may be easily recognized and suitable treatment by rachicentesis instituted, and therefore its employment in the routine physical examination of a patient with poliomyelitis is of the utmost importance.

10. Since an attempt has been made in this article to introduce a distinctly new clinical entity in reference to poliomyelitis, these descriptions and reports should be read in order to obtain the essential points. 